PROCEEDINGS OF THE

AMERICAN MATHEMATICAL SOCIETY

Volume 137, Number 8, August 2009, Pages 2773-2783

S 0002-9939(09)09851-7

Article electronically published on February 3, 2009

\title{
COMPUTATIONAL UNSOLVABILITY OF DOMAINS OF ATTRACTION OF NONLINEAR SYSTEMS
}

\author{
NING ZHONG
}

(Communicated by Julia Knight)

\begin{abstract}
Let $S$ be the domain of attraction of a computable and asymptotically stable hyperbolic equilibrium point of the non-linear system $\dot{x}=f(x)$. We show that the problem of determining $S$ is computationally unsolvable. We also present an upper bound of the degree of unsolvability of this problem.
\end{abstract}

\section{Preliminaries}

One of the central objects of study in continuous dynamical systems is the domain of attraction of an asymptotically stable equilibrium point. Determining this domain is one of the most important problems in (Lyapunov) stability theory. In the late 1960's there was a surge of theoretical studies analyzing properties of such domains. In recent years much effort has been devoted to the development of numerical methods for the estimation of these domains, which has resulted in numerous numerical algorithms. In contrast, relatively little theoretical work on computability of these domains exists. In fact, it is not yet known whether or not domains of attraction of computable systems are computable.

In this paper, we show that, despite the existence of various successful numerical estimations, the problem of determining domains of attraction is computationally unsolvable. We also present an upper bound of the degree of unsolvability of the problem by showing that the domains of attraction of computable systems are recursively enumerable. Speaking roughly, a set is computable if its image can be generated by a computer with arbitrary precision.

For real computation, there are several non-equivalent models. Among them are the BSS model [3] and the Turing-machine-based bit model [18, 14, 8, 13, 15], [11, and [19]. The BSS model permits real numbers as input and uses infiniteprecision arithmetic, while the bit model operates on infinite sequences of bits and uses finite-precision arithmetic; that is, "bit approximations" to the output are computed with arbitrary precision if good "bit approximations" to the input are accessible. Since finite-precision is generally used in "practical computation," we use the bit model in this paper. Associated with the bit model is the Type-Two Theory of Effectivity (TTE) [19].

Received by the editors July 11, 2008, and, in revised form, December 8, 2008.

2000 Mathematics Subject Classification. Primary 03D80; Secondary 34D45, 68Q17.

Key words and phrases. Recursive open/closed subsets of $\mathbb{R}^{n}$, r.e. open/closed subsets of $\mathbb{R}^{n}$, computable functions, continuous dynamical systems, domain of attraction of an asymptotically stable equilibrium point. 
In TTE, every point $x \in \mathbb{R}^{n}$ is represented by a sequence $\left\{r_{k}\right\}$ of points with rational coordinates satisfying $\left|x-r_{k}\right|<2^{-k}$. Any such sequence $\phi: \mathbb{N} \rightarrow \mathbb{Q}^{n}$, $\phi(k)=r_{k}$, is called a $\rho^{n}$-name of $x$. A $\rho^{n}$-name is called computable if it is generated by a Turing machine without input. A point $x$ in $\mathbb{R}^{n}$ is called computable if it has a computable $\rho^{n}$-name. The same class of computable points is defined if points of rational coordinates are replaced by points of dyadic rational coordinates. A rational number is called a dyadic rational if it is of the form $\frac{j}{2^{m}}$. A matrix is computable if every entry of the matrix is a computable real or complex number. The norm of an $n \times n$ matrix $A$ is defined as the operator norm: ||$A||=\sup _{|x| \neq 0}|A x| /|x|=\max _{|x|=1}|A x|$, where $|x|=\sqrt{x_{1}^{2}+\ldots+x_{n}^{2}}, x \in \mathbb{R}^{n}$.

Definition 1.1 (Recursive and recursively enumerable open subsets of $\mathbb{R}^{n},[19$ ).

(1) An open subset $U \subseteq \mathbb{R}^{n}$ is called recursively enumerable (r.e.) open if there is a computable sequence $\left\{a_{k}\right\}$ of points with rational coordinates in $\mathbb{R}^{n}$ and a computable sequence $\left\{r_{k}\right\}$ of positive rational numbers such that $U=\bigcup_{k=0}^{\infty} B\left(a_{k}, r_{k}\right)$, where $B\left(a_{k}, r_{k}\right)=\left\{x \in \mathbb{R}^{n}:\left|x-a_{k}\right|<r_{k}\right\}$.

(2) A closed subset $K \subseteq \mathbb{R}^{n}$ is called r.e. closed if there is a Turing machine that enumerates all rational open balls intersecting $K$.

(3) An open subset $U \subseteq \mathbb{R}^{n}$ is called recursive (also called computable) open if $U$ is r.e. open and its complement is r.e. closed.

Roughly speaking, an open subset $U$ of $\mathbb{R}^{n}$ is r.e. if there is a computer program that sketches the image of $U$ by plotting rational open balls, which will eventually fill up $U$. We may not know how well these balls are filling up $U$ if $U$ is merely r.e. On the other hand, if $U$ is recursive, then there is a program that plots the balls filling $U$ up to precision $2^{-k}$ (in terms of Hausdorff distance) on input $k$ ([19]).

Definition 1.2 (Computable functions, [1]). A function $f: A \rightarrow \mathbb{R}^{m}, A \subseteq \mathbb{R}^{n}$, is computable if there is an oracle Turing machine $M$ such that, for each $x \in A$ and any dyadic $\rho^{n}$-name $\phi$ of $x, M$ computes with oracle $\phi$ a dyadic rational point $r_{k}$ satisfying $\left|f(x)-r_{k}\right| \leq 2^{-k}$ upon input $k \in \mathbb{N}$.

This definition leads naturally to (the "non-uniform" concept of) polynomialtime computability of Ker-I Ko.

Definition 1.3 (Polynomial-time computable functions, [11). A function $f: \mathbb{R}^{n} \rightarrow$ $\mathbb{R}^{m}$ is called polynomial-time computable if there exist an oracle Turing machine $M$ that computes $f$ and a polynomial $p: \mathbb{N} \rightarrow \mathbb{N}$ such that for any $l \in \mathbb{N}$, any $x \in\left[-2^{l}, 2^{l}\right]^{n}$ (the Cartesian product of $n$ copies of $\left[-2^{l}, 2^{l}\right]$ ), and any $\rho^{n}$-name $\phi$ of $x$ with $\phi(k)=\left(d_{k}^{1}, d_{k}^{2}, \ldots, d_{k}^{n}\right)$ and $d_{k}^{j} \in D_{k}=\left\{\alpha \cdot 2^{-k}: \alpha \in \mathbb{Z}\right\}$ for $1 \leq j \leq n$, one has $T_{M}(\phi, l) \leq p(l)$, where $T_{M}(\phi, l)$ is the number of moves for $M$ to halt on input $l$ with oracle $\phi$.

Next we briefly review notions necessary for defining the domain of attraction. Consider a non-linear autonomous system

$$
\dot{x}=f(x(t)),
$$

where $\dot{x}=d x / d t$ and $f: E \rightarrow \mathbb{R}^{n}$ ( $E$ is an open subset of $\mathbb{R}^{n}$ ) is continuously differentiable on $E$ ([5]).

Definition 1.4 (Stable and asymptotically stable equilibrium points, 9]). Let $x\left(t, x_{0}\right)$ denote the solution to (1.1) corresponding to the initial condition $x(0)=x_{0}$. 
(i) A point $x_{0}$ is called an equilibrium point of (1.1) if $f\left(x_{0}\right)=0$. An equilibrium point $x_{0}$ is called hyperbolic if none of the eigenvalues of the gradient matrix $D f\left(x_{0}\right)$ of $f$ at $x_{0}$ has zero real part.

(ii) An equilibrium point $x_{0}$ of (1.1) is called stable if for any $\epsilon>0$, there exists a $\delta>0$ such that $\left|x(t, \tilde{x})-x_{0}\right|<\epsilon$ for all $t \geq 0$, provided $\left|\tilde{x}-x_{0}\right|<\delta$. Furthermore, $x_{0}$ is called asymptotically stable if it is stable and there exists a (fixed) $\delta_{0}>0$ such that $\lim _{t \rightarrow \infty} x(t, \tilde{x})=x_{0}$ for all $\tilde{x}$ satisfying $\left|\tilde{x}-x_{0}\right|<\delta_{0}$.

If $x_{0}$ is an equilibrium point of (1.1), then the constant solution $x\left(t, x_{0}\right) \equiv x_{0}$ is the unique solution to the problem $\dot{x}=f(x)$ and $x(0)=x_{0}$. It is also known that a hyperbolic equilibrium point $x_{0}$ is asymptotically stable if all eigenvalues of $D f\left(x_{0}\right)$ have negative real parts $\left.(9]\right)$.

Definition 1.5 (Domain of attraction, 9]). Suppose that $x_{0}$ is an asymptotically stable equilibrium point of the system (1.1). Then the domain of attraction (or basin of attraction or region of asymptotical stability) of $x_{0}$ is defined by

$$
S=\left\{\tilde{x} \in E: x(t, \tilde{x}) \rightarrow x_{0} \text { as } t \rightarrow \infty\right\} .
$$

The domain of attraction $S$ is an open subset of $\mathbb{R}^{n}$. If $E$ is simply connected, then so is $S$. Furthermore, if $S$ is bounded, then its boundary is formed by whole trajectories (that is, solution curves); in particular, $S$ is bounded by (unstable) equilibrium point(s) in the one-dimensional case ([ $[\underline{6}$ and the references therein).

\section{Computational unsolvability of domains of Attraction}

Theorem 2.1. There exists a $C^{\infty}$ (infinitely differentiable) and polynomial-time computable function $g: \mathbb{R}^{2} \rightarrow \mathbb{R}$ such that the following system

$$
\left\{\begin{array}{l}
\dot{x}=x g(x, y)-y \\
\dot{y}=y g(x, y)+x
\end{array}\right.
$$

has a unique equilibrium point at $(0,0)$. This unique equilibrium point is hyperbolic and asymptotically stable, and the domain of attraction of $(0,0)$ is non-computable.

Proof. It is easy to see that $(0,0)$ is the only equilibrium point of the system (2.1). Denote the right-hand sides of the two equations in (2.1) as $h_{1}$ and $h_{2}: h_{1}(x, y)=$ $x g(x, y)-y$ and $h_{2}(x, y)=y g(x, y)+x$. The function $g$ is to be constructed such that $(0,0)$ is an asymptotically stable hyperbolic equilibrium point of (2.1) and the domain of attraction $S$ of $(0,0)$ is a disk centered at $(0,0)$ with a non-computable radius. This shows that $S$ is non-computable and completes the proof.

We construct a function $f: \mathbb{R} \rightarrow \mathbb{R}$, and then we set $g(x, y)=f\left(x^{2}+y^{2}\right)$. The function $f$ satisfies the following properties:

(i) $f: \mathbb{R} \rightarrow \mathbb{R}$ is $C^{\infty}$ and polynomial-time computable;

(ii) $f$ decreases in a neighborhood of $0, f(w)=0$ for all $\alpha \leq w \leq 2$, and $f(w)<0$ for all $w \in[0, \infty) \backslash[\alpha, 2]$,

where $\alpha=\sum_{n=0}^{\infty} 2^{-a(n)}$, and $a: \mathbb{N} \rightarrow \mathbb{N}$ is a computable one-to-one function that enumerates a r.e. but non-recursive set $A$. It is known that $\alpha$ is a non-computable real number ([15]). Without loss of generality assume that $0 \notin A$ and $1 \in A$. Thus $1 / 2<\alpha<2$. Let $\phi: \mathbb{N} \rightarrow \mathbb{D}, \phi(n)=\sum_{j=0}^{n} 2^{-a(j)}$, where $\mathbb{D}$ is the set of dyadic rational numbers. Since $a: \mathbb{N} \rightarrow \mathbb{N}$ is computable, it follows that $\phi$ is a computable function. Let $\gamma=2^{-(a(0)+1)}$. Then $0<\gamma<1 / 2$. Let $M$ be a Turing machine 
computing the function $\phi$, and let $t(n)$ be the total number of moves for $M$ to run on inputs $0,1, \ldots, n$.

The function $f$ to be constructed will be a superposition of countably many "V-shaped hats", hanging below and suspended at the endpoints of each interval $(\gamma, \phi(n))$. We take the following $C^{\infty}$ function as our standard V-shaped hat:

$$
\varphi(w)= \begin{cases}-e^{-w^{2} /\left(1-w^{2}\right)} & \text { for }|w|<1 \\ 0 & \text { for }|w| \geq 1\end{cases}
$$

This hat is $\mathrm{V}$-shaped with half-width 1 , depth 1 , centered at 0 , and is hanging below $[-1,1]$. Also $\varphi \in C^{\infty}(\mathbb{R})$ is easily seen to be polynomial-time computable. Define the $n$-th hat $\varphi_{n}(w)$ hanging below the interval $(\gamma, \phi(n))$ by the formula

$$
\varphi_{n}(w)=\frac{d_{n}}{2} \varphi\left(\frac{w-c_{n}}{d_{n}}\right)
$$

where $d_{n}=\frac{\phi(n)-\gamma}{2}$ and $c_{n}=\frac{\phi(n)+\gamma}{2}$. Then $\varphi_{n}$ is a V-shaped hat of depth $d_{n} / 2$ $\left(d_{n} / 2\right.$ is bounded by 1 since $\left.d_{n} \leq 2\right)$, half-width $d_{n}$, and centered at $c_{n}$. In addition, we make two more special hats $\varphi_{-1}$ and $\varphi_{\infty}$ defined by the following formulae:

$$
\varphi_{-1}(w)= \begin{cases}-e^{-\frac{(8 w-1)^{2}}{9-(8 w-1)^{2}}} & \text { for }-\frac{1}{4}<w<\frac{1}{2} \\ 0 & \text { otherwise }\end{cases}
$$

and

$$
\varphi_{\infty}(w)= \begin{cases}-e^{-\frac{1}{w-2}} & \text { if } w>2 \\ 0 & \text { otherwise. }\end{cases}
$$

The hat $\varphi_{-1}$ is hanging below $\left(-\frac{1}{4}, \frac{1}{2}\right)$ with center at $1 / 8$ and has the property that $\varphi_{-1}(0)<0$ and $\varphi_{-1}$ decreases on the interval $\left(-\frac{1}{4}, \beta\right)$, where $\beta=\min \{1 / 8, \gamma\}$. The function $\varphi_{\infty}$ is a half-hat hanging below $(2, \infty)$. Now we are ready to give a description of the function $f: \mathbb{R} \rightarrow \mathbb{R}$,

$$
f(w)=\varphi_{-1}(w)+\varphi_{\infty}(w)+\sum_{n=0}^{\infty} 2^{-t(n)} \varphi_{n}(w) .
$$

It is readily seen that $f$ is $C^{\infty}$ in $\mathbb{R}$ and has the property (ii). (The main observation we need for checking infinite differentiability of $f$ is that $1 / d_{n}=2 /(\phi(n)-\gamma) \leq$ $2 /(\phi(0)-\gamma)=2^{a(0)+2}$ for all $n$.) An argument similar to Ko's proofs of Theorem 3.1 and Theorem 6.2 [1] shows that $f$ is polynomial-time computable. Set $g(x, y)=$ $f\left(x^{2}+y^{2}\right)$. Obviously $g$ is $C^{\infty}$ and polynomial-time computable. Since the gradient matrix $D\left(h_{1}, h_{2}\right)$ of $\left(h_{1}, h_{2}\right)$ has the following form:

$$
D\left(h_{1}, h_{2}\right)=\left(\begin{array}{ll}
\partial h_{1} / \partial x & \partial h_{1} / \partial y \\
\partial h_{2} / \partial x & \partial h_{2} / \partial y
\end{array}\right)=\left(\begin{array}{ll}
f(r)+2 x^{2} D f(r) & 2 x y D f(r)-1 \\
2 x y D f(r)+1 & f(r)+2 y^{2} D f(r)
\end{array}\right)
$$

where $r=x^{2}+y^{2}$, it follows that $D\left(h_{1}, h_{2}\right)(0,0)=\left(\begin{array}{cc}f(0) & -1 \\ 1 & f(0)\end{array}\right)$. It is easy to see that $f(0)+i$ and $f(0)-i$ are the eigenvalues of $D\left(h_{1}, h_{2}\right)(0,0)$, and both eigenvalues have negative real part $f(0)$, which shows that $(0,0)$ is an asymptotically stable hyperbolic equilibrium point of (2.1). We claim that the disk $D_{\alpha}=\{(x, y) \in$ $\left.\mathbb{R}^{2}: x^{2}+y^{2}<\alpha\right\}$ is the domain of attraction of $(0,0)$. To see this, we first reduce the system (2.1) to a single equation. By multiplying the first equation in (2.1) by 
$x$ and the second by $y$, then adding the two resulting equations, we can reduce the system (2.1) to the following equation:

$$
\dot{r}=2 r f(r) \text {. }
$$

It is easy to see that if $\left(x\left(t, x_{0}, y_{0}\right), y\left(t, x_{0}, y_{0}\right)\right)$ is the solution to the system (2.1) satisfying the initial conditions $x(0)=x_{0}$ and $y(0)=y_{0}$, and if $(\gamma, \delta)$ is the maximal interval of existence of this solution, then $r\left(t, r_{0}\right)=x^{2}\left(t, x_{0}, y_{0}\right)+y^{2}\left(t, x_{0}, y_{0}\right)$ is the solution of (2.2) satisfying the initial condition $r(0)=r_{0}=x_{0}^{2}+y_{0}^{2}$ with $(\gamma, \delta)$ as its maximal interval of existence.

We now show that $S \subseteq D_{\alpha}$. For any $\left(x_{0}, y_{0}\right)$ satisfying $\alpha \leq x_{0}^{2}+y_{0}^{2}=$ $r_{0} \leq 2$, let $\left(x\left(t, x_{0}, y_{0}\right), y\left(t, x_{0}, y_{0}\right)\right)$ be the solution to the system (2.1) satisfying the initial conditions $x(0)=x_{0}$ and $y(0)=y_{0}$. Then $r\left(t, r_{0}\right)=x^{2}\left(t, x_{0}, y_{0}\right)+$ $y^{2}\left(t, x_{0}, y_{0}\right)$ is the solution of (2.2) satisfying the initial condition $r(0)=r_{0}$. Since $f\left(r_{0}\right)=0$, the solution $r\left(t, r_{0}\right)$ is a constant solution that is equal to $r_{0}$ for all $t$. This implies that $x^{2}\left(t, x_{0}, y_{0}\right)+y^{2}\left(t, x_{0}, y_{0}\right)=r_{0}$ for all $t$. Consequently, $\lim _{t \rightarrow \infty}\left(x\left(t, x_{0}, y_{0}\right), y\left(t, x_{0}, y_{0}\right)\right) \nrightarrow(0,0)$. Since $S$ is simply connected and $(0,0) \in$ $S \cap D_{\alpha}$, this shows that $S \subseteq D_{\alpha}$.

Next we show that $D_{\alpha} \subseteq S$. First we observe that the problem $\dot{r}=2 r f(r)$ and $r(0)=0$ has the constant solution $r(t, 0) \equiv 0$, while the problem $\dot{r}=2 r f(r)$ and $r(0)=\alpha$ has the constant solution $r(t, \alpha) \equiv \alpha$. Now for any $\left(x_{0}, y_{0}\right) \in D_{\alpha} \backslash\{(0,0)\}$, let $r_{0}=x_{0}^{2}+y_{0}^{2}$; then $0<r_{0}<\alpha$. If $\delta$, the right endpoint of the maximal existence interval $(\gamma, \delta)$ of the solution $\left(x\left(t, x_{0}, y_{0}\right), y\left(t, x_{0}, y_{0}\right)\right)$, is not $\infty$, then $\lim _{t \rightarrow \delta^{-}} r\left(t, r_{0}\right)=\infty$. Thus there exists a $t_{0}$ such that $0<t_{0}<\delta$ and $r\left(t_{0}, r_{0}\right)=$ $\alpha$. Due to the uniqueness of the solution, the solution $r\left(t, r_{0}\right)$ coincides with the constant solution $r(t, \alpha) \equiv \alpha$. This is a contradiction because $r_{0}<\alpha$. Therefore, $\delta=\infty$. It remains to show that $\lim _{t \rightarrow \infty} r\left(t, r_{0}\right)=0$. Otherwise, suppose that $\lim _{t \rightarrow \infty} r\left(t, r_{0}\right)=\beta \neq 0$ or $r\left(t, r_{0}\right)$ oscillates without a limit as $t \rightarrow \infty$. We note that $0<r\left(t, r_{0}\right)<\alpha$ for all $t \geq 0$, for otherwise $r\left(t, r_{0}\right)$ is either identically 0 or identically $\alpha$, which is false because $0<r\left(0, r_{0}\right)=r_{0}<\alpha$. Since $0<r_{0}<\alpha$ and $f(r)<0$ on $[0, \alpha)$, it follows that $\left.\frac{d r}{d t}\right|_{t=0}=2 r_{0} f\left(r_{0}\right)<0$; hence $r\left(t, r_{0}\right)$ decreases near $t=0$. Thus if $r\left(t, r_{0}\right)$ turns around becoming increasing at a later time, then there is a $t_{1}>0$ such that $0<r\left(t_{1}, r_{0}\right)<\alpha$ and $2 r\left(t_{1}, r_{0}\right) f\left(r\left(t_{1}, r_{0}\right)\right)=\left.\frac{d r}{d t}\right|_{t_{1}}=0$, which implies that $f\left(r\left(t_{1}, r_{0}\right)\right)=0$. This contradicts the fact that $f(r)<0$ for all $0<r<\alpha$. Therefore $r\left(t, r_{0}\right)$ is decreasing for all $t \geq 0, \lim _{t \rightarrow \infty} r\left(t, r_{0}\right)$ exists, and $\lim _{t \rightarrow \infty} r\left(t, r_{0}\right) \leq r_{0}$. Now if $\lim _{t \rightarrow \infty} r\left(t, r_{0}\right)=\beta \neq 0$, then $r=\beta$ is an asymptote of the trajectory $r=r\left(t, r_{0}\right)$ and it follows that $\lim _{t \rightarrow \infty} d r\left(t, r_{0}\right) / d t=0$. Thus, $2 \beta f(\beta)=2 \lim _{t \rightarrow \infty} r\left(t, r_{0}\right) f\left(r\left(t, r_{0}\right)\right)=\lim _{t \rightarrow \infty} d r\left(t, r_{0}\right) / d t=0$ with $0<\beta \leq r_{0}<$ $\alpha$, so it follows that $f(\beta)=0$ with $0<\beta \leq r_{0}<\alpha$, which is false. This shows that $\lim _{t \rightarrow \infty} r\left(t, r_{0}\right)=0$ for any $0<r_{0}<\alpha$. In other words, $\lim _{t \rightarrow \infty} x\left(t, x_{0}\right)=0$ and $\lim _{t \rightarrow \infty} y\left(t, y_{0}\right)=0$ for all $\left(x_{0}, y_{0}\right) \in D_{\alpha}$. The proof is now complete.

The above theorem shows that if the dimension is higher than one, then the domain of attraction of a computable asymptotically stable equilibrium point of a computable system is not necessarily computable, even in the case where the system has exactly one equilibrium point and this unique equilibrium point is hyperbolic. For a computable system in one-dimensional space, if the system has only finitely many equilibrium points, then the domain of attraction of any asymptotically stable equilibrium point is computable. 
Proposition 2.2. Let $f: E \rightarrow \mathbb{R}$ be a computable function that is also locally Lipschitz, where $E$ is a computable open interval of $\mathbb{R}$ or $E=\mathbb{R}$. Assume that $f$ has finitely many roots in $E$. Then the domain of attraction, $S$, of any asymptotically stable equilibrium point of (1.1) is a computable open interval.

Proof. It is known that if a computable function has only finitely many roots, then each of its roots is computable ([11, 19]). On the other hand, $S$ is an interval that is either unbounded or has unstable equilibrium point(s) as its bound(s) ([6]). Therefore, $S$ is a computable interval.

Remark 2.3. If $f$ has infinitely many roots, then the conclusion of Proposition 2.2 no longer holds. For example, let $f$ be the function constructed in Theorem 2.1. Then $f$ is $C^{\infty}$ and polynomial-time computable. It is easy to verify that 0 is an asymptotically stable equilibrium point of the system $\dot{x}=f(x)$ and $(-1 / 4, \alpha)$ is the corresponding domain of attraction, which is non-computable.

If we further assume that $f$ is polynomial-time computable in Proposition 2.2 , is $S$ also polynomial-time computable? The following proposition gives a strong negative answer. Not only is $S$ non-polynomial-time computable, but there is no complexity bound on $S$.

Proposition 2.4. For any computable real number $c, 1 / 2<c<1$, there exists a polynomial-time computable function $f$ defined on $\mathbb{R}$ such that 0 is an asymptotically stable equilibrium point of the system $\dot{x}=f(x)$ and $(-c, c)$ is the domain of attraction of 0 .

Proof. By Theorem 4.4 of [11], there exists a strictly increasing polynomial-time computable function $f_{1}$ on $[1 / 2,1]$ such that $c$ is the unique root of $f_{1}$. Let $f_{2}(x)=-f_{1}(-x), x \in[-1,-1 / 2]$. Then $f_{2}$ is a strictly increasing polynomialtime computable function on $[-1,-1 / 2]$ such that $-c$ is the unique root of $f_{2}$. In particular, $f_{1}(1 / 2)=-f_{2}(-1 / 2)=b, b<0$ is a polynomial-time computable real number. Define the desired function $f$ as follows:

$$
f(x)= \begin{cases}f_{2}(-1) & x<-1 \\ f_{2}(x) & x \in[-1,-1 / 2) \\ 2 b x & x \in[-1 / 2,1 / 2) \\ f_{1}(x) & x \in[1 / 2,1) \\ f_{1}(1) & x \geq 1 .\end{cases}
$$

Since $f: \mathbb{R} \rightarrow \mathbb{R}$ is computable and globally Lipschitz, the system $\dot{x}=f(x)$ has a unique computable solution for any initial value $x_{0} \in \mathbb{R}([7])$. Moreover, because $f(0)=0$ and $f^{\prime}(0)=2 b<0, x=0$ is an asymptotically stable equilibrium point of $\dot{x}=f(x)$. A proof similar to the proof of Theorem 2.1 shows that the domain of attraction of 0 is $(-c, c)$.

If $f$ is a polynomial-time computable analytic function, then all roots of $f$ are polynomial-time computable ([1] $)$. This fact together with Proposition 2.2 leads to the following corollary.

Corollary 2.5. Let $f: \mathbb{R} \rightarrow \mathbb{R}$ be a polynomial-time computable analytic function. Assume that $x_{0} \in \mathbb{R}$ is an asymptotically stable equilibrium point of (1.1). Then the domain of attraction of $x_{0}$ is a polynomial-time computable open interval. (Recall that an open interval $(\alpha, \beta)$ is called polynomial-time computable if $\alpha$ and $\beta$ are polynomial-time computable real numbers.) 


\section{Domains of ATtraction of COMPUtABle SyStems ARE R.E. OPEN}

Although the domain of attraction $S$ of a computable and asymptotically stable hyperbolic equilibrium point of a computable system may not be computable, it is r.e. open, as Theorem 3.1 below shows. This indicates that $S$ can be approximated from "inside", starting with a small neighborhood containing the equilibrium point and lying inside $S$. However, since $S$ may not be computable, there may not exist any algorithm that can provide information on how far an approximation is from filling up $S$.

Throughout this section we assume that the system $\dot{x}=f(x)$ defines a dynamical system on $\mathbb{R}^{n}$; that is, for any $x_{0} \in \mathbb{R}^{n}$, the unique solution $x\left(t, x_{0}\right)$ to the problem

$$
\dot{x}=f(x), \quad x(0)=x_{0}
$$

is defined for all $t \in \mathbb{R}$. Then the solution map $x: \mathbb{R} \times \mathbb{R}^{n} \rightarrow \mathbb{R}^{n},\left(t, x_{0}\right) \mapsto$ $x\left(t, x_{0}\right)$ (also written as $\left.x\left(t, x_{0}\right)=x_{t}\left(x_{0}\right)\right)$ has the following properties: $x_{s+t}\left(x_{0}\right)=$ $x_{s}\left(x_{t}\left(x_{0}\right)\right), x_{-t}\left(x_{t}\left(x_{0}\right)\right)=x_{0}$, and $x_{t}\left(x_{-t}\left(x_{0}\right)\right)=x_{0}$ for all $s, t \in \mathbb{R}$ and $x_{0} \in \mathbb{R}^{n}$ $([9])$.

Theorem 3.1. Let $f: \mathbb{R}^{n} \rightarrow \mathbb{R}^{n}$ be a $C^{1}$-computable function (meaning that both $f$ and $D f$ are computable). Assume that $x_{0}$ is a computable and asymptotically stable hyperbolic equilibrium point of (3.1). Let $S$ be the domain of attraction of $x_{0}$. Then $S$ is a r.e. open subset of $\mathbb{R}^{n}$.

Proof. First we note that under the assumption that $f$ is $C^{1}$-computable, the solution map $x: \mathbb{R} \times \mathbb{R}^{n} \rightarrow \mathbb{R}^{n}$ is computable $([7])$.

Since the system (3.1) is autonomous, without loss of generality, we may assume that $x_{0}$ is the origin 0 . An outline of the proof proceeds as follows. A small ball $B$ is computed so that $B$ is centered at the origin and contained in the domain of attraction $S$. Then $B$ is expanded "effectively" to fill $S$. The expansion is done by letting points in $B$ flow backward in time. For the purpose of construction of $B$, we write $f(x)=A x+F(x)$, where $A=D f(0)$ and $F(x)=f(x)-A x$. Clearly, if $x(t, a)$ is the solution of the following integral equation

$$
x(t, a)=e^{t A}\left(a+\int_{0}^{t} e^{-s A} F(x(s, a)) d s\right)=e^{t A} a+\int_{0}^{t} e^{(t-s) A} F(x(s, a)) d s,
$$

then it is also the solution of the initial value problem $\dot{x}=f(x(t))$ and $x(0)=a$ as is seen by differentiation (an application of the product rule and the fundamental theorem of calculus), where $e^{t A}=\sum_{k=0}^{\infty} t^{k} A^{k} / k$ ! and $d e^{t A} / d t=A e^{t A}$. In the following, we compute an upper bound for $\left\|e^{t A}\right\|$ from eigenvalues of $A$, and then we use this upper bound to compute a radius for the desired ball $B$.

Since $F(x)=f(x)-A x$, it follows that $F(0)=0, D F(0)=0, F$ and $D F$ are both computable for $f$ and $D f$ are computable functions. Thus there is a computable function $d: \mathbb{N} \rightarrow \mathbb{N}$ such that

$$
|F(x)-F(y)| \leq 2^{-m}|x-y| \text { whenever }|x| \leq 2^{-d(m)} \text { and }|y| \leq 2^{-d(m)} .
$$

Since 0 is an asymptotically stable hyperbolic equilibrium point, all eigenvalues of the $n \times n$ matrix $A=D f(0)$ have negative real parts ([9]). Moreover, since $D f$ is computable, all entries in the matrix $A$ are computable; consequently, the coefficients of the characteristic polynomial $\operatorname{det}\left(A-\lambda I_{n}\right)$ of $A$ are computable, where $\operatorname{det}\left(A-\lambda I_{n}\right)$ denotes the determinant of $A-\lambda I_{n}$ and $I_{n}$ is the $n \times n$ unit matrix. Thus all eigenvalues of $A$ are computable, for they are zeros of the computable 
polynomial $\operatorname{det}\left(A-\lambda I_{n}\right)$. Assume that $\lambda_{1}, \lambda_{2}, \ldots, \lambda_{n}$ are eigenvalues of $A$ (counting multiplicity). Then a rational number $\alpha>0$ can be computed from the $\lambda_{k}$ 's such that $\operatorname{Re}\left(\lambda_{k}\right)<-\alpha$ for $1 \leq k \leq n$, where $\operatorname{Re}\left(\lambda_{k}\right)$ denotes the real part of $\lambda_{k}$. We break $\alpha$ into two parts for later use: Let $\alpha_{1}$ and $\alpha_{2}$ be two rational numbers such that $0<\alpha_{1}<\alpha$ and $\alpha_{1}+\alpha_{2}=\alpha$.

Next we construct a simple closed curve in the complex plane that contains all eigenvalues of $A$ in its interior. Let $M$ be a natural number such that $M>$ $\max \{\alpha, 1\}$ and $\max \left\{\left|\lambda_{k}\right|: 1 \leq k \leq n\right\} \leq M-1$, and let $\Gamma=\Gamma_{1} \cup \Gamma_{2} \cup \Gamma_{3} \cup \Gamma_{4}$ be the boundary of a rectangular region in $\mathbb{R}^{2}$, where $\Gamma_{1}=\{(x, y): x=-\alpha,-M \leq$ $y \leq M\}, \Gamma_{2}=\{(x, y):-M \leq x \leq-\alpha, y=M\}, \Gamma_{3}=\{(x, y): x=-M,-M \leq$ $y \leq M\}$, and $\Gamma_{4}=\{(x, y):-M \leq x \leq-\alpha, y=-M\}$. Then $\Gamma$ encloses all eigenvalues of $A$ in its interior (with the counterclockwise direction as the positive direction). We observe that for any $\xi \in \Gamma$, the matrix $A-\xi I_{n}$ is invertible and $\operatorname{det}\left(A-\xi I_{n}\right)=\prod_{k=1}^{n}\left(\lambda_{k}-\xi\right)$.

Claim 1. The function $g: \Gamma \rightarrow \mathbb{R}, g(\xi)=\left\|\left(A-\xi I_{n}\right)^{-1}\right\|$, is computable, where $\left(A-\xi I_{n}\right)^{-1}$ is the inverse of the matrix $A-\xi I_{n}$.

Proof of Claim 1. Since $\left(A-\xi I_{n}\right)^{-1} \mapsto\left\|\left(A-\xi I_{n}\right)^{-1}\right\|$ is computable, it suffices to show that $\xi \mapsto\left(A-\xi I_{n}\right)^{-1}$ is computable. For each $1 \leq i, j \leq n$, let $M_{i j}$ be the determinant of the matrix obtained by deleting the $i$ th row and $j$ th column of $A-\xi I_{n}$, and let $C_{i j}=(-1)^{i+j} M_{i j}$. Then each $C_{i j}$ is a polynomial in $\xi$ with computable coefficients (which are sums of products of elements of $A$ ). Now let $b_{i j}$ denote the $i j$ th-entry of $\left(A-\xi I_{n}\right)^{-1}$. Then $b_{i j}=C_{j i} / \operatorname{det}\left(A-\xi I_{n}\right)$ (see, for example, Appendix I [9]), which is computable from $A$ and $\xi$. This completes the proof of Claim 1.

Since $g: \Gamma \rightarrow \mathbb{R}$ is computable and $\Gamma$ is computably compact, the maximum of $g$ on $\Gamma, \max _{\xi \in \Gamma}\left\|\left(A-\xi I_{n}\right)^{-1}\right\|$, is computable ([19]). Let $K_{1} \in \mathbb{N}$ be an upper bound of this computable maximum. Now for any $t \geq 0$, from (5.47) and Problem 5.19 of Chapter 1, Paragraph 5 of [10], $e^{t A}=-\frac{1}{2 \pi i} \int_{\Gamma} e^{t \xi}\left(A-\xi I_{n}\right)^{-1} d \xi$. A simple calculation shows that $\left\|-\frac{1}{2 \pi i} \int_{\Gamma_{j}} e^{t \xi}\left(A-\xi I_{n}\right)^{-1} d \xi\right\| \leq K_{1} M e^{-\alpha t} / \pi, 1 \leq j \leq 4$. Let $K=4 M K_{1}$. Then

$$
\left\|e^{t A}\right\| \leq \sum_{j=1}^{4}\left\|-\frac{1}{2 \pi i} \int_{\Gamma_{j}} e^{t \xi}\left(A-\xi I_{n}\right)^{-1} d \xi\right\| \leq K e^{-\alpha t}, \text { for all } t \geq 0 .
$$

Next we compute an integer $m$ such that $2^{-m} \leq \frac{\alpha_{2}}{2 K}$, and then set $r=2^{-d(m)} /(2 K)$ and $B=B(0, r)=\left\{a \in \mathbb{R}^{n}:|a|<r\right\}$, where the computable function $d$ is as in (3.3). In the following claim, we show that this ball $B$ is what we desire.

Claim 2. For any $a \in B$, if $x(t, a)$ is the solution of (3.2), then $\lim _{t \rightarrow \infty} x(t, a)=0$. Therefore, $B \subseteq S$.

Proof of Claim 2. For $a \in B$ and $t \geq 0$, define

$$
x^{(0)}(t, a)=0, \quad x^{(j)}(t, a)=e^{t A} a+\int_{0}^{t} e^{(t-s) A} F\left(x^{(j-1)}(s, a)\right) d s, \quad j \geq 1 .
$$


If the two inequalities

$$
\begin{gathered}
\left|x^{(j)}(t, a)-x^{(j-1)}(t, a)\right| \leq K|a| e^{-\alpha_{1} t} / 2^{j-1}, \\
\left|x^{(j)}(t, a)\right| \leq 2^{-d(m)} e^{-\alpha_{1} t},
\end{gathered}
$$

hold for all $j \in \mathbb{N}$, then it is clear from (3.4) that $\left\{x^{(j)}(t, a)\right\}_{j=1}^{\infty}$ is a Cauchy sequence that converges to the solution of (3.2); that is, $\lim _{j \rightarrow \infty} x^{(j)}(t, a)=x(t, a)$, uniformly for all $t \geq 0$ and $a \in B$. Furthermore, (3.5) shows that $|x(t, a)| \leq 2^{-d(m)} e^{-\alpha_{1} t}$ for all $t \geq 0$ and $a \in B$, which in turn implies that $\lim _{t \rightarrow \infty} x(t, a)=0$ for all $a \in B$. Thus the proof is complete if we can show that (3.4) and (3.5) hold for all $j \in \mathbb{N}$. We employ an induction argument to prove this fact. Since $\left|x^{(1)}(t, a)-x^{(0)}(t, a)\right|=$ $\left|e^{t A} a\right| \leq|| e^{t A}|| \cdot|a| \leq K e^{-\alpha t}|a| \leq K e^{-\alpha_{1} t}|a|$ and $\left|x^{(1)}(t, a)\right|=\left|e^{t A} a\right| \leq\left\|e^{t A}\right\| \cdot|a| \leq$ $K e^{-\alpha t} \cdot 2^{-d(m)} / 2 K \leq 2^{-d(m)} e^{-\alpha_{1} t}$, the two inequalities hold for $j=1$. Assume that the induction hypotheses hold for $k \leq j$. Then for $k=j+1$, since

$$
\begin{aligned}
\left|x^{(j+1)}(t, a)-x^{(j)}(t, a)\right| & =\left|\int_{0}^{t} e^{(t-s) A}\left(F\left(x^{(j)}(s, a)\right)-F\left(x^{(j-1)}(s, a)\right)\right) d s\right| \\
& \leq \int_{0}^{t}\left\|e^{(t-s) A}\right\| \cdot\left|F\left(x^{(j)}(s, a)\right)-F\left(x^{(j-1)}(s, a)\right)\right| d s \\
& \leq \int_{0}^{t} K e^{-\alpha(t-s)} \cdot 2^{-m}\left|x^{(j)}(s, a)-x^{(j-1)}(s, a)\right| d s \\
& \leq 2^{-m} \int_{0}^{t} K e^{-\alpha(t-s)} \cdot \frac{K|a| e^{-\alpha_{1} s}}{2^{j-1}} d s \\
& =2^{-m} \frac{K^{2}|a|}{2^{j-1}} e^{-\left(\alpha_{1}+\alpha_{2}\right) t} \int_{0}^{t} e^{\alpha_{2} s} d s \\
& \leq 2^{-m} \frac{K^{2}|a| e^{-\alpha_{1} t}}{\alpha_{2} \cdot 2^{j-1}} \leq \frac{\alpha_{2}}{2 K} \cdot \frac{K^{2}|a| e^{-\alpha_{1} t}}{\alpha_{2} \cdot 2^{j-1}}=\frac{K|a| e^{-\alpha_{1} t}}{2^{j}}
\end{aligned}
$$

(recall that $2^{-m} \leq \alpha_{2} / 2 K$ ), (3.4) holds for $j+1$. Furthermore, from the above inequality and the induction hypothesis, we obtain that

$$
\left|x^{(j+1)}(t, a)\right| \leq\left|x^{(j)}(t, a)\right|+\frac{K|a| e^{-\alpha_{1} t}}{2^{j}} \leq \sum_{k=0}^{j} \frac{K|a| e^{-\alpha_{1} t}}{2^{k}} \leq 2 K|a| e^{-\alpha_{1} t} \leq \frac{e^{-\alpha_{1} t}}{2^{d(m)}} .
$$

It follows that (3.5) holds for $j+1$. Thus (3.4) and (3.5) hold for all $j \in \mathbb{N}$. This completes the proof of Claim 2.

Finally we expand $B$ to fill $S$ by letting points in $B$ flow backward in time. Let $\left\{r_{k}\right\}$ be a computable sequence that enumerates all non-negative rational numbers. Since the solution map $x: \mathbb{R} \times \mathbb{R}^{n} \rightarrow \mathbb{R}^{n},(t, a) \mapsto x(t, a)$, is computable, the sequence $\left\{x_{r_{k}}(a)\right\}$ is a computable sequence of functions from $\mathbb{R}^{n}$ to $\mathbb{R}^{n}$ (recall that $\left.x_{r_{k}}(a)=x\left(r_{k}, a\right)\right)$. By Theorem 6.2.4 of [19], the open set $x_{-r_{k}}(B)=x_{r_{k}}^{-1}(B)$ is recursively enumerable for all $k \in \mathbb{N}$. Thus the set $\bigcup_{k \in \mathbb{N}} x_{-r_{k}}(B)$ is a r.e. open subset of $\mathbb{R}^{n}$. We show that $S=\bigcup_{k \in \mathbb{N}} x_{-r_{k}}(B)$, which completes the proof of the theorem. To see this, we first observe that for any $a \in S$, since $\lim _{t \rightarrow \infty} x_{t}(a)=0$ and $x_{t}(a)=x(t, a)$ is continuous in $t$, there exists a rational number $r_{k}>0$ such that $b=x_{r_{k}}(a) \in B$. Then $a=x_{-r_{k}}(b) \in x_{-r_{k}}(B)$ and consequently, $S \subseteq$ $\bigcup_{k \in \mathbb{N}} x_{-r_{k}}(B)$. On the other hand, for any $a \in \bigcup_{k \in \mathbb{N}} x_{-r_{k}}(B)$, there exist $k \in \mathbb{N}$ and $b \in B$ such that $a=x_{-r_{k}}(b)$, and so $x_{r_{k}}(a)=b$. Then $\lim _{t \rightarrow \infty} x(t, a)=$ $\lim _{t \rightarrow \infty} x_{t-r_{k}}\left(x_{r_{k}}(a)\right)=\lim _{t \rightarrow \infty} x_{t-r_{k}}(b)=0$, which implies that $a \in S$. 
We end this section with an open question: Is the domain of attraction (DA) of an asymptotically stable equilibrium point of a polynomial planar system $\dot{x}=$ $P(x, y)$ and $\dot{y}=Q(x, y)$ computable? Here both $P$ and $Q$ are real polynomials with computable coefficients. In the case where the DA is bounded, it is known that the boundary of the DA is formed by either a limit cycle or a phase polygon. We also mention in passing that to decide an upper bound for the number of limit cycles in the above system and to investigate their relative positions is the second part of Hilbert's 16th problem, which is unresolved.

\section{ACKNOWLEDGMENT}

The author thanks the anonymous referee for helpful comments and suggestions.

\section{REFERENCES}

[1] O. Aberth. Computable analysis and differential equations. Intuitionism and Proof Theory, edited by A. Kino, J. Myhill and R.E. Vesley, Studies in Logic and the Foundations of Mathematics, pp. 47-52, North-Holland, Amsterdam, 1970. MR.0276087 (43:1835)

[2] O. Aberth. The failure in computable analysis of a classical existence theorem for differential equations. Proc. Amer. Math. Soc., Vol. 30, pp. 151-156, 1971. MR0302982 (46:2124)

[3] L. Blum, M. Shub and S. Smale. On a theory of computation and complexity over the real numbers: NP-completeness, recursive functions and universal machines. Bull. Amer. Math. Soc., Vol. 21, No. 1, pp. 1-46, 1989. MR974426(90a:68022)

[4] M. Braverman and M. Yampolsky. Non-computable Julia sets. J. Amer. Math. Soc., Vol. 19, No. 3, pp. 551-578, 2006. MR2220099 (2007m:37110)

[5] E. Coddington and N. Levinson. Theory of Ordinary Differential Equations. International Series in Pure and Applied Mathematics, McGraw-Hill Book Company, New York-TorontoLondon, 1955. MR0069338 (16:1022b)

[6] R. Genesio, M. Tartaglia, and A. Vicino. On the estimation of asymptotic stability regions: State of the art and new proposals. IEEE Transactions on Automatic Control, Vol. AC-30, No. 8, pp. 747-755, August 1985. MR794208 (86g:93046)

[7] D.S. Graça, N. Zhong and J. Buescu. Computability, noncomputability and undecidability of maximal intervals of IVPs. To appear in Transactions of the AMS.

[8] A. Grzegorczyk. Computable functionals. Fund. Math., Vol. 42, pp. 168-202, 1955. MR0086756 (19:238b)

[9] M. Hirsch and S. Smale. Differential equations, dynamical systems, and linear algebra. Pure and Applied Mathematics, Vol. 60, Academic Press, New York-London, 1974. MR0486784 (58:6484)

[10] T. Kato. Perturbation theory for linear operators. Die Grundlehren der mathmatischen Wissenschaften in Einzeldarstellungen, Band 132, Springer-Verlag, New York-Berlin, 1966. MR0203473 (34:3324)

[11] K. Ko. Complexity Theory of Real Functions. Progress in Theoretical Computer Science. Birkhäuser, Boston, 1991. MR1137517 (93i:03057)

[12] K. Ko. Computational complexity of fractals. Proceedings of the 7th and 8th Asian Logic Conferences, pp. 252-269, Singapore Univ. Press, Singapore, 2003. MR2051982 (2005a:03123)

[13] D. Lacombe. Extension de la notion de fonction récursive aux fonctions d'une ou plusieurs variables réelles. III. Comptes Rendus de l'Académie des Sciences Paris, Vol. 241, pp. 151153, 1955. MR0072080 (17:225e)

[14] S. Mazur. Computable Analysis. Rozprawy Mat., Vol. 33, PWN, 1963. MR0153553 (27:3517)

[15] M. B. Pour-El and J. I. Richards. Computability in Analysis and Physics. Springer, Berlin, 1989. MR1005942 (90k:03062)

[16] M. B. Pour-El and N. Zhong. The wave equation with computable initial data whose unique solution is nowhere computable. Math. Logic Quarterly, Vol. 43, No. 4, pp. 499-509, 1997. MR 1477618 (98m:03097)

[17] R. Rettinger and K. Weihrauch. The computational complexity of some Julia sets. Proceedings of the Thirty-Fifth Annual ACM Symposium on Theory of Computing, pp. 177-185, ACM, New York, 2003. MR2121048(2005j:68060) 
[18] A. M. Turing. On computable numbers, with an application to the Entscheidungsproblem. Proc. London Math. Soc., Vol. 2, No. 42, pp. 230-265, 1936.

[19] K. Weihrauch. Computable Analysis. Springer, Berlin, 2000. MR1795407(2002b:03129)

[20] K. Weihrauch and N. Zhong. Is wave propagation computable or can wave computers beat the Turing machine? Proc. London Math. Soc., Vol. 85, No. 3, pp. 312-332, 2002. MR 1912053 (2003i:03064)

[21] K. Weihrauch and N. Zhong. An algorithm for computing fundamental solutions. SIAM Journal on Computing, Vol. 35, No. 6, pp. 1283-1294, 2006. MR2217146 (2007f:03104)

[22] N. Zhong and K. Weihrauch. Computability theory of generalized functions. Journal of ACM, Vol. 50, Issue 4, pp. 469-505, 2003. MR2146883 (2006d:03111)

Department of Mathematical Sciences, University of Cincinnati, Cincinnati, Ohio 45221-0025

E-mail address: Ning.Zhong@uc.edu 